\title{
A Wake up Call
}

\section{for Sleep Disordered Breathing}

\section{Guest Editorial}

\author{
S. N. Jain \\ Camp U.S.A. \\ 208 Robb Lane \\ Greens Berg \\ P. A. 15601 \\ U.S.A.
}

Sleep occupies $25-40 \%$ of our lives, yet fails to receive an appropriate amount of attention in medical practice. Over the past 20 years there has been an increasing awareness of the role that sleep plays in health throughout the day. Sleep-related breathing disorder (SRBD) in particular, is often not suspected clinically and are difficult to diagnose confidently.

SRBD is a group of conditions involving abnormalities of breathing during sleep that range from mild snoring, often made worse by sedative drugs or alcohol ingestion, to severe obstructive sleep apnea syndrome (OSAS) with severe derangement of sleep architecture and secondary organ dysfunction. The prevalence of SRBD is not accurately known. But estimates have put the prevalence at approximately $1-4 \%$ of the general North American population (1). SRBD results in disturbance of sleep architecture secondary to frequent arousals. This can result in daytime hyper somnolence, impaired cognitive function, fatigue and insomnia. Patients with OSAS are at increased risk for motor vehicular accidents having more than twice as many accidents as contro!s (2). Systemic hypertension is a well-dncumented association of heavy snoring and OSAS (3).

The natural history of SRBD is not accurately known. Patients with OSAS who are not adequately treated have a significant mortality. The potential causes of mortality are varied and include the cardiovascular complications of systemic and pulmonary hypertension and incidents related to excessive daytime somnolence.

The clinical presentation of SRBD is subtle and could be easily missed unless sought for. Snoring is one of the prime symptoms of OSAS, but is accepted as normal unless very loud or intrusive. The symptoms of snoring, hyper somnolence, early morning headaches, fatigue, poor concentration and pcor sleep are often overlooked by the patient and the clinician. These symptoms may be embarrassing or are attributed to laziness or ageing. Many patients may be treated for hypertension and cardiac failure without the true etiologic cause being suspected. Increased public and physician awareness is key to making the diagnosis of SRBD. 
Suspicion of the diagnosis of SRBD requires confirmation as the clinical evaluation alone is not adequate. Subjective impression identifies only $52 \%$ of patients with OSAS with a specificity of $70 \%$ (4). Most patients show no abnormality while awake, making it necessary to study sleeping patients. Nocturnal polysomnography (PSG), which involves recording of sleep staging and respiratory variables, is currently the gold standard for accurately diagnosing and assessing the severity of OSAS. PSG however, requires the time of medical and paramedical staff and is an expensive technology.

To overcome the expense and inconvenience of PSG, other approaches to diagnosis have been studied. CT scanning of the upper airway during wakefulness and sleep have demonstrated significant changes in soft tissue volume in the pharynx in OSAS, but these findings are insufficient to confirm the diagnosis (5).

Pulse oximetry to measure arterial oxygen saturation is a widely employed noninvasive screening test for OSAS. It has a sensitivity of $80 \%$ and a negative predictive value of $87 \%$ in establishing the presence of OSAS (6). The addition of a clinical score that is determined by the presence of heavy snoring, interrupted breathing, daytime hyper somnolence, obesity and hypertension can further improve the negative predictive value.

\section{Treatment of OSAS}

There are a number of effective strategies available for the treatment of OSAS. The initial approach in most individuals involves behaviour modification that includes avoiding nocturnal sedatives / alcohol and in obese patients, weight reduction. Compliance with weight loss is however, poor.

Pharmacological approaches are attractive because of the ease of administration. Tricyclic antidepressants appear effective in some cases of OSAS by suppressing rapid eye movement sleep and improving upper airway coordination. Significant side-effects (cardiac arrhythmias and urinary retention) and limited efficacy has prevented their widespread use. Nocturnal oxygen administration has been shown to prevent severe falls in oxygen saturation during sleep although daytime sleepiness and hemodynamic parameters are unaffected (7).

\section{Mechancial Methods}

A number of devices have beeen described for the mechanical relief of OSAS. These range from nightly insertion of a nasopharyngeal airway to intra-oral dental prosthesis or tongue retaining devices. Oral appliances that reposition the lower jaw may be helpful for the retrognathic patient, provided the nasal airway is patent and bruxism does not occur in sleep. These have the advantages for low cost and are not particularly invasive. Patient acceptance is generally poor and results are mixed, although good results have been observed in mild OSAS (8). Only a few large enough studies have been performed that establish the patient characteristics predictive of success.

The current accepted treatment of choice for OSAS is nasal continuous positive airway pressure (CPAP). This consists of a firmly fitting nasal mask attached to an air compressor with the pressure in the system maintained at a sufficient level to prevent collapse of the upper airway during respiration in sleep. CPAP has been shown to improve survival (9), reduce blood pressure, reduce nocturnal falls in arterial oxygen saturation, improve cardiac function and decrease daytime hyper somnolence. Reported side-effects include feelings of claustrophobia and local facial and nasal irritation. The patient compliance with CPAP is about $45-68 \%$.

\section{Surgical Treatment}

Surgical treatment is widely practiced as this holds out the hope of prolonged resolution. The results of a number of surgical procedures are, however, disappointing. Tracheostomy bypasses the site of obstruction and is effective in abolishing apneic episodes and improving mortality in virtually all cases. Tracheostomy is often poorly tolerated due to problems of recurrent local and chest infections, secretions and psychosocial maladjustment. It is currently reserved only to the severed cases as a means of ensuring survival while weight loss and other definitive therapy is instituted.

Nasal surgery to relieve nasal obstruction and tonsillectomy to remove large tonsils in adults may be useful in some individuals with mild to moderate OSAS. Nasal surgery may also be needed to facilitate the later use of CPAP. 
Surgical revision of the soft palate and oropharyngeal tissues (UPPP) has been widely used for the management of OSAS. UPPP has been shown to be very effective in abolishing snoring but the outcome is not very satisfactory in OSAS. Objective improvemnt in OSAS has been found in between $40-66 \%$ of patients undergoing this procedure as airway obstruction may persist within the trans palatal or the hypopharyngeal airway (10). Extensive reconstruction of the upper airway by maxillary mandibular and hyoid advancement in patients with hypopharngeal-base of tongue obstruction can result in significant improvement.

There have been at least 175 papers dealing with the surgical management sleep apnea and snoring. However, only about 54 of these articles have been observed to have performed with reasonable study design. The rest of the articles were hampered by a low study population ( less than nine subjects), lack of objective postoperative evaluation or were just review articles. Statistically, there were no differences in the preoperative clinical characteristics between the responders and non-responders to UPPP, although the non-responders tended to have worse OSAS than the responders. When the site of the pharyngeal obstruction was identified preoperatively, and even though several different techniques were used to identify the site of obstruction, results of UPPP improved to $74.6 \pm 27 \%$ compared with $22.8 \pm 29 \%$ in patients with isolated retroglossal or a combined retro palatal and retroglossal obstruction (10). Minimal information was available on long-term follow-up. The considerable postoperative problems and the potential for late complications coupled with the lack of a guaranteed favourable outcome have meant that UPPP as a procedure is undertaken only after careful consideration. Results of retroglossal surgery are hard to analyse as these procedures were usually performed in patients who were UPPP failures or were performed simultaneously with UPPP.

There were many drawbacks to analysis of these articles (10). Randomized, controlled studies of the surgical treatment of OSAS did not exist. Follow-up data reported in most studies were incomplete and short-term in nature. Reporting of pre and post operative PSG data was non-uniform. In addition, inclusion and exclusion criteria and the surgical procedures varied widely across the studies.
A final evaluation of the role of pharyngeal surgery in the treatment of OSAS is not available. Further research is needed to answer the following questions: Should the preoperative determination of the pharyngeal site of obstruction be done in sleep or is an office procedure such as upper endoscopy with the Muller maneuver be adequate ? If a relapse occurs in OSAS patients undergoing pharyngeal surgery, is further surgery beneficial and at what site?

Laser-assisted uvulopalatoplasty (LAUP) is an out patient surgical procedure performed in the office setting with the patient under local anesthesia involving three to five laser sessions. Treatments involve a progressive enlargement of the oropharyngeal airway by trimming and reshaping the uvula and the posterior soft palate. The sessions are performed 4-6 weeks apart, allowing healing between each session and retraction of the uvula and palate tissue secondary to scarring. The results of the LAUP procedures involve removal of significantly less tissue than UPPP.

There has been a remarkable proliferation of LAUP procedure without an objective documentation of its efficacy. Snoring, being an extremely common disorder and its perception as a nagging nuisance has unfortunately, offered a unique opportunity for the LAUP (given its promotion as a simple an innocuous treatment for snoring) to undergo this proliferation. The public has come to request, and sometimes even demand laser treatment for snoring, and there is a potential for inappropriate use by health care professionals with little training in the field of sleep disordered breathing. In conjunction with this promotion, LAUP has subtly been expanded by many practitioners to treat OSAS. Recent guidelines from the American Sleep Disorders Association have recommended that LAUP not be performed in OSAS as adequate peer-reviewed data do not exist to its effectiveness and that the patient be objectively evaluated preoperatively for sleep related respiratory disturbances (11). Patients should be informed that the risks benefits and complications of LAUP have not been established. They should also be informed of incurring a delay in the diagnosis of OSAS because snoring, a primary diagnostic symptom of OSAS, may be obviated by this surgical procedure. 


\section{Conclusions}

OSAS is the most common and best-recognized form of SRBD. Effective treatment is available with CPAP being the treatment of choice, once the essential nonspecific measures of excluding endocrine and other treatable causes, including weight loss and avoiding sedative agents have been undertaken. The expense of CPAP apparatus and the need to use this for prolonged period may prompt some patients to evaluate other sub-optimal options. Surgical treatment will continue to be a gamble or a treatment of last resort. Whatever plan is developed, it is important that the patient be reassessed after six months and then by annual PSGs.

The potential population of snorer / apneic patients is so wide and the cost of the proper care so high that the field is ripe for abuses. The confusion between symptoms of snoring and the incidence of SRBD has compounded this proliferation. Cut-rate care that cuts corners is often more expensive in the long run. Interested readers should be aware of the potential inappropriate applications of surgical treatment, especially LAUP, and be alerted to the best medical interests of their patients.

\section{References}

1. Young T, Palta M, Dempsey J et al. (1993): The occurrence of sleep-disordered breathing among middle-aged adults $N$ Engl J Med; 328 : 1230-35.

2. George CF, Nickerson PW, Hanly PG et al (1987): Sleep apnea patients have more automobile accidents. Lancet , ii (8556) : 447.

3. Carlson JT, Hedner JA, Ejnell Het al. (1994): High prevalence of hypertension in sleep apnea patıents independent of obesity. Am. Respir Crit Care Med, 150 - 72-77.

4. Viner S, Szalat JP, Joffstein V. (1991) Are history and physical examination a good screening test for sleep apnea. Ann Intern Med ; $115: 2414-18$.

5 Pepin $J L$, Ferretti G, Veale D et al. (199I). Somnofluoroscopy, computed tomography and cephalometry in the assessment of the airway in obstructive sleep apnea. Thorax, 47, 150-6.

6. Farney RJ, Walker LE, Jensen RL et al. (1986) : Ear oxtmetry to detect apnea and differenttate rapid eye movement and non-raptd eye movement sleep. Chest ; $89: 533-39$.

7. Fletcher EC, Munafo DA. (1990) : Role of nocturnal oxygen therapy in obstructive sleep apnea Chest, 98: 14971504.

8. Schmidt-Nowara $W$, Lowe A, Wiegand L et al (1995) An American Sleep Disorders Association review : Oral appliances for the treatment of snoring and obstructive sleep apnea Sleep ; 18 (6) : 501-510.

9. He J, Kryger MH, Zorick FJ et al. (1988) - Mortality and apnea index in obstructive sleep apnea Chest, 94 : $531-38$.

10. Sher A, Schectmen K, Piccirillo J. (1996): An American Sleep Disorders Association review : the efficacy of surgical modifications of the upper airway in adults with obstructive sleep apnea syndrome. Sleep; 19 156-77

11. An American Sleep Disorders Association Report. Practice Parameters for the use of Laser-assisted Uvulopalatoplasty. (1994) Sleep, $17(8) \cdot 744-48$. 